\title{
Automated Modelling of Masonry Walls: a ML and AR Approach
}

\author{
Pierpaolo D'Agostino \\ Federico Minelli
}

Abstract

A methodology for the automated delineation of brick masonries from images to a vector representation is discussed in this paper. Python environment is chosen for the coding activity in order to provide automation to the process. Edge detection and vector delineation of brick joints are followed by a phase of brick clustering for masonry classification. The implementation of the process is tested on a video sequence to simulate an augmented reality application for masonry detection.

Keywords

masonry delineation, Canny algorithm, AR, digital survey.
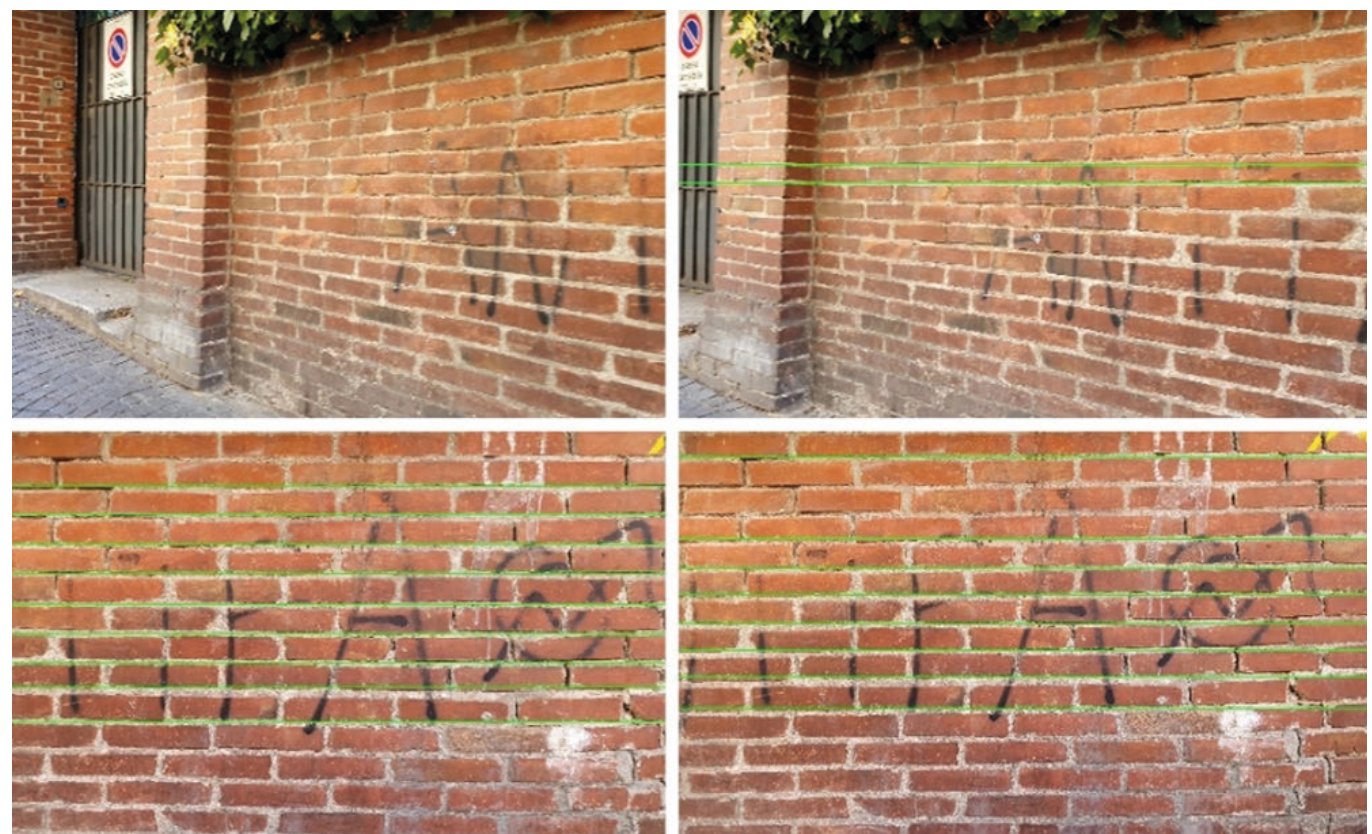


\section{Introduction}

Parametric and algorithmic modeling are constantly becoming increasingly useful tools for the automation of processes destined, among other things, to the management and use of cultural heritage, in particular taking into account the digital evolution that the tools for its recovery and restoration have had, opening up to the implementation of techniques such as machine learning and augmented reality. Particularly in the Italian context, in fact, it is known how vast is the heritage worthy of attention for its redevelopment and restoration, both for historical buildings and for modernist buildings that also have valuable characteristics. Clearly, it is in this direction that the new frontiers of digital surveying are evolving by the possibility of describing the artifacts through their "digital twins" and by their direct use rather than for the realization of classic technical drawings.

Digital survey is often indicated as a starting point for the creation of structured data models. In fact, nowadays their use in reverse engineering processes is stable, but still partly subject to manual modeling (according to the interpretation of a human being).

In this spirit of participation in the automation of such processes, we are working on automated workflows for the digitization and interactive use of historical and cultural heritage that exploit machine learning applications to obtain cognitive feedback in real time from the architectural artefact. To this end, augmented reality applications, running on handheld devices used as virtual reality interfaces, can mimic the human presence in space and provide a computer-generated model, in a virtual space, modeled on the real context, with which the users can interact.

The research in progress, taking advantage of an approach specifically base on scripting, concerns the digitization of masonry, a so often required specification that could be very complex to reach as automatic outcome, especially in historic buildings where different construction techniques can be implemented, to extract adequate information to classify and model the underlying structure (fig. I).

Fig. I. Real cases of exposed masonry: Cellammare Palace (on the left) and the 'Sferisterio' in Fuorigrott (on the right) in Naples.
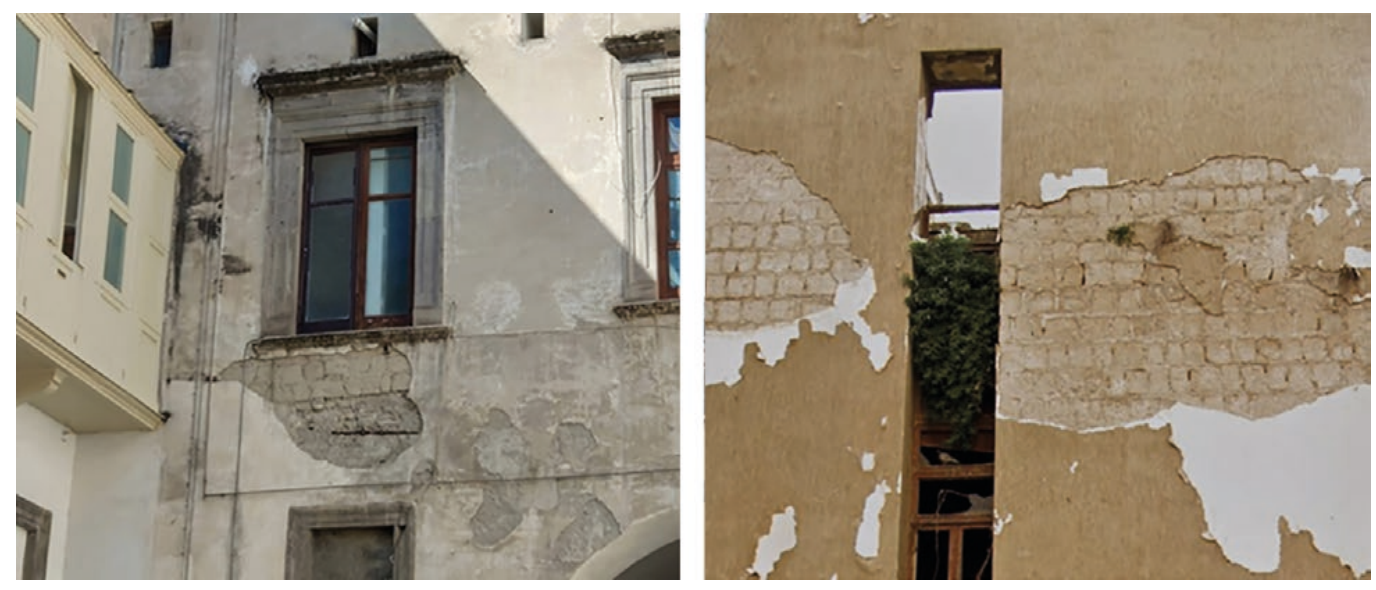

The delineation based on images or video streams of the masonry, in particular, is centered on "edge detection" to identify the key areas of the masonry structure: firstly, the regions where the joints between the bricks are located. This is a fundamental step for the masonry morphology classification process as it allows the segmentation of single bricks.

To obtain a vector representation of the masonry, in order to achieve a referred 3D modeling, it is crucial to define horizontal and vertical joints between the bricks, detected and delineated from the contours. These operations are pointed to find the intersection points between the lines in order to outline the contours of each brick and, then, to evaluate the area of each closed boundary used to classify and reconstruct the masonry structure. Machine learning techniques come into play in this classification phase and in particular, an approach based on data clustering is implemented for the recognition of bricks with faces of different dimensions exposed. The methodological approach is described below. 
The ability to carry out this operation in real time and in an unsupervised way opens up the integration of the model thus obtained with augmented reality systems. Multi-channeling through the use of augmented reality thus finds an interesting development path with high added value since the quality and quantity of data acquired and processed to support the interaction enrich the experience of interaction with the monument by placing it in an ideal context: real-time feedback of the architecture. The fundamental innovation, in fact, regardless of the scale dimensions of the displays (tablets, smartphones) is the activation of the development of an integrated interaction project which, by exploiting augmented reality, enhances communication and knowledge, managing to simplify and make the interaction with architecture more complete and engaging, raising the level of attention (fig. 2).

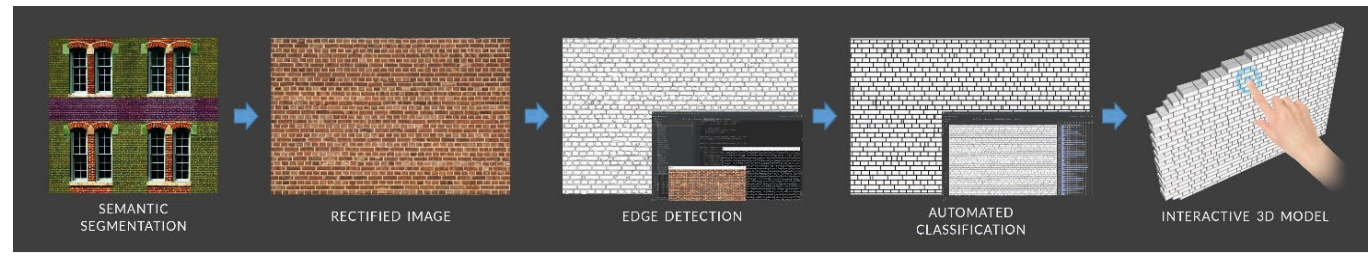

MASONRY CLASSIFICATION
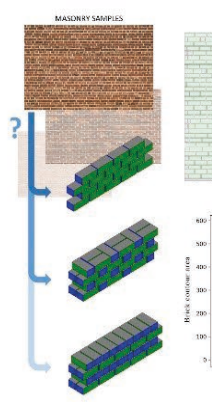
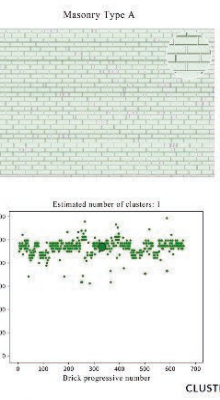

$\&$

REAL TIME RECOGNITION

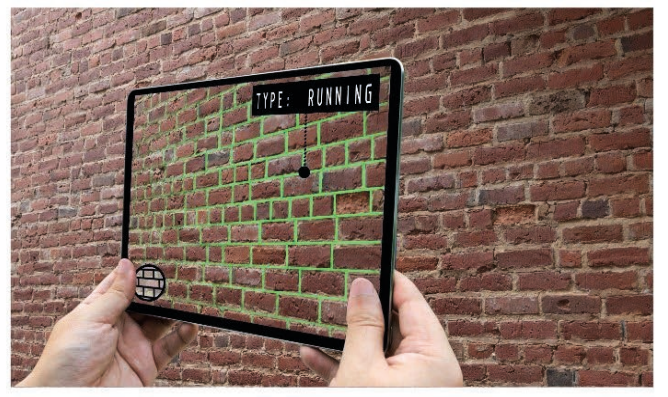

\section{Methodology}

What we would describe here is a workflow for automatic detection and modeling of periodic walls. We started by considering how to apply parametric and algorithmic modeling in such automation processes used to take.

The possibility in objective processes that allow automatically to pass from the point cloud to parametric models is still very rare, for example in Building Information Modeling approaches, for which the possibility of identifying, for example, openings or automatically segmenting building components is still an arduous challenge. Or, for example, in the models intended for the detailed analysis of its parts (for example FEM analysis), of those characteristics that make up the individual construction components.

Starting from simulated data but corresponding to possible applications starting from real data, the process leads to the production of solid models of brick walls. The theoretical approach aims to be applied in several city models applications. Procedural city modeling ranges from the digital reconstruction of entire neighborhoods to the production of single building models [Zhu et al. 20 I 6]. From the analysis of available studies on digital reconstruction of urban areas using algorithmic procedures [Musialski et al. 20 I3] and automated asbuilt modeling [Patraucean et al. 20 I5] our modeling approach can be evaluated with these methods. The usage of images and point clouds for the recognition and modeling of architectural features are of great interest and in continuous development. The automation of this parametric architecture modeling process from on-site survey materials is also a very active field in scientific research today [Czerniawski and Leite 2020]. However, differently by standardized situations, the morphology of building walls can be very complex, especially in historic buildings, but several techniques can be implemented to extract adequate information to classify and model the underlying structure. The image-based delineation of masonry, in particular, relies on edge detection to identify key areas of the masonry structure: first, 
the regions where the gaps between the bricks are located. This is a fundamental step for the masonry morphology classification process as it allows the segmentation of single bricks, but the subsequent 3D modeling phase also requires an investigation as it allows to obtain a full reconstruction of the asset [D'Agostino \& Minelli 2020].

Moreover, this work focuses on the automatic detection of masonry from images and its interaction in AR applications. To answer to the goal of optimization of the ongoing investigation in this connection between AR acquiring process and a future-proof real time processing, with the need to test several outcomes, an image-based approach to vectorization of wall textures characterized by horizontal rows is delineated and tested on different masonry samples. The programming language Python is selected, for its flexibility and effectiveness for code writing activities in order to achieve process automation, for the coding activities to achieve the automation of the process.

The work specifically aims to digitally reconstruct the masonry walls based exclusively on rectified images. As anticipated, the approach proposed first seeks to achieve a vector representation of each brick in respect of the real masonry texture. The second purpose, rarely addressed in the literature before, is to create an interactive delineation of masonry on the basis of the current arrangement of the bricks. The approach tries to be effective on masonry made by bricks of a single size.

Segmentation and three-dimensionality for the reconstruction of the individual bricks is tested on several images of masonry to verify the consistency of the proposed workflow to the unpredictable conditions that can occur when dealing with real masonry. The RGB source images are transformed into the HSV color representation in the Canny algorithm for the first brick edge detection. The binary image of the edges is used to extract the contours of the brick. In order to obtain a vector representation of the masonry, the UV coordinates of the edges of the horizontal brick joints are first detected and delineated by the contours. The vertical joints are detected in a second step and added to the previous one, respecting the height of each row of bricks. The intersection points between the lines allow to outline the contours of each brick. The area value of each closed contour is calculated to classify and reconstruct the masonry structure.

Mean Shift algorithm is used to sort the outlines of the front bricks by the outlines of the side facing ones. The quantity of clusters detected and the number of occurrences of front and side bricks allows to classify each masonry analyzed in one of the 3 categories investigated in this work (fig. 3). Finally, a reconstruction of the 3D mesh of the masonry is performed and the geometric model is saved in a DXF file.

A data clustering approach is implemented for recognizing bricks with faces of different sizes exposed.The Mean Shift clustering algorithm is then used to separate the oriented bricks with the front face in view from the side ones, based on the area value of their respective boundaries. The front facing bricks, in fact, show a lower value of the boundary area, while the side facing bricks are characterized by a greater value of the boundary area. Choosing the Mean
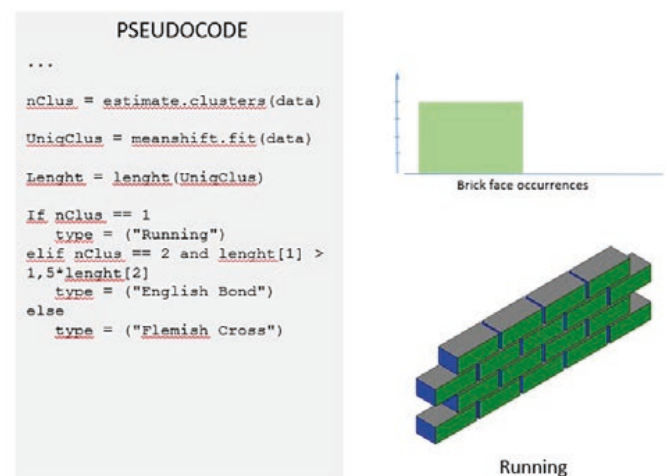

Running
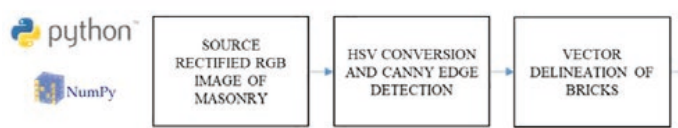

\section{Classification Tree}
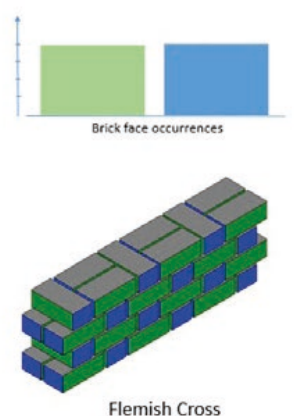

Flemish Cross
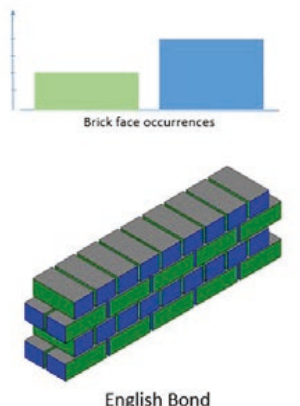

English Bond

CONTOUR AREA MAMPUINEG AND BRICK
CLUSTERING 
Shift clustering algorithm relies on its ability to directly estimate the number of clusters from the dataset. In fact, other clustering algorithms require the user to manually specify the cluster number file to search for in the dataset. The masonry investigated in this study, however, range from types in which a single cluster of bricks is found to types in which two clusters occur. Deepening the possibility to join AR visual acquisition with the described workflow, we propose that the vectorization of walls' joints could pass for their identification directly on visual flows. So, the approach studied for raster images is applied to video sequences in order to allow a real time detection and vectorization of the masonry. An augmented reality application is therefore simulated on a video stream, acquired in real circumstances (fig. in cover page). An effective delineation of horizontal rows is obtained with robust outcomes on several frames of the video stream. This opens to the complete vectorization of the masonry in real time, that will be addressed in future studies.

\section{Conclusions}

Multi-channeling through the use of augmented reality thus finds an interesting development path with high added value because the quality and quantity of data designed to support the interaction enrich the experience by placing it in an ideal context: when needed and to whom really need.

The fundamental issue, in fact, is not so many the scale dimensions of the displays (megascreen, tablet, installation, smartphone) that make the difference, as the development of an integrated communication project which, by exploiting augmented reality, enhances communication by managing to simplify and make the interaction more complete and engaging, raising the level of attention.

Segmentation and three-dimensionality applied in wall digitation is tested on a video of the masonry to verify the consistency of the proposed workflow to the unpredictable conditions that can occur when dealing with real masonry.

The results obtained in the study can be applied in architecture surveys and fruition application to establish a direct connection between the captured image and the reconstructed geometry. Practical applications of this procedure can also be found in the automated BIM modeling process for 3D reconstruction of buildings from survey material and in the automated FEM modeling of masonry structures, as it can provide a detailed model of masonry morphology. Future work should consist of further experimentation on different and more complex masonry structures to obtain results of wider applicability. The masonry classification, already attained with images, should be also tested on video sequences.

\section{References}

Czerniawski Thomas, Leite Fernanda (2020). Automated digital modeling of existing buildings: A review of visual object recognition methods. In Automation in Construction. Elsevier, 113 , pp. 103-131.

D'Agostino Pierpaolo, Minelli Federico (2020). Automated image-based detection and vectorization of coursed masonry for parametric modeling of building envelope. In Marchiafava Veronica, Picollo Marcello (eds). Colour and Colorimetry Multidisciplinary Contributions. Milano: Gruppo del Colore - Associazione Italiana Colore, pp. 43-50.

Musialski Przemyslaw, Wonka Peter, Aliaga Daniel G., Wimmer Michael,Van Gool Luc, Purgathofer Werner (20 I3). A survey of urban reconstruction. In Computer Graphics Forum, 32 (6), pp. I 46- 177.

Patraucean Viorica, Armeni Iro, Nahangi Mohammad, Yeung Jamie, Brilakis loannis, Haas Carl (20I5). State of research in automatic as-built modelling. In Advanced Engineering Informatics, 29 (2), pp. $162-171$.

Zhu Wenhua, Wang Dexian, Eynard Benoit, Bricogne Matthieu, Remy Sebastien (2016). Procedural approach for 3D modeling of city buildings. In IFIP Advances in Information and Communication Technology, 467, pp. I 37-I 48.

\footnotetext{
Authors

Pierpaolo D'Agostino, Dept. of Civil, Building and Environmental Engineering, University of Naples Federico II, pierpaolo.dagostino@unina.it Federico Minelli, Dept. of Industrial Engineering, University of Naples Federico II, federico.minelli@unina.it
} 
